\title{
Scarless excision of an insertion sequence restores capsule production and virulence in Acinetobacter baumannii
}

\author{
Clémence Whiteway ${ }^{1,2}$, Adam Valcek (D) ${ }^{1,2}$, Chantal Philippe $^{3}$, Mojca Strazisar ${ }^{4,5}$, Tim De Pooter $^{4,5}$, Ivan Mateus ${ }^{6}$, Anke Breine ${ }^{1,2}$ and $^{1,2}$ \\ Charles Van der Henst iD ${ }^{1,2}{ }^{凶}$
}

(c) The Author(s) 2022

We identify a new mechanism mediating capsule production and virulence in the WHO and CDC priority ESKAPE pathogen Acinetobacter baumannii. Non-capsulated and avirulent bacteria can revert into a capsulated and virulent state upon scarless excision of an ISAba13 insertion sequence under stress conditions. Reversion events fully restore capsule production and in vivo virulence. This increases our knowledge about A. baumannii genome dynamics, and the regulation of capsule production, virulence and resistance.

The ISME Journal (2022) 16:1473-1477; https://doi.org/10.1038/s41396-021-01179-3

\section{INTRODUCTION}

Acinetobacter baumannii is an opportunistic human pathogen and a constant growing threat because of its propensity to aquire multidrug resistance [1]. For this ESKAPE pathogen [2], ranked as critical priority by WHO [3] and CDC [4], new antimicrobial strategies for prophylaxis and treatment are urgently needed. Despite its clinical relevance, only little is known about $A$. baumannii overall virulence and its regulation [5, 6]. However, production of envelope determinants including the exopolysaccharide capsule is critical to escape the host immune system, to resist desiccation and antimicrobial treatments [6]. Genes required for biosynthesis and export of exopolysaccharides are clustered within the capsule locus (K-locus). Capsule composition and structure are highly variable between $A$. baumannii isolates. So far, at least 128 different K-locus types [7] have been identified and over $40 \mathrm{~K}$ units structure have been elucidated [8]. In addition to the high genetic diversity amongst isolates, phenotypic heterogeneity is generated in clonal populations by a high frequency phenotypic phase variation mechanism, impacting capsule production in $A$. baumannii which allows interconversion between virulent (VIR-O) and avirulent bacteria (AV-T) [9-12].

Here, we identify a new mechanism that controls the virulence and resistance of $A$. baumannii, which modulates capsule formation by the insertion/excision of the insertion sequence (IS) element ISAba13. Our study therefore contributes to an increased knowledge about genome plasticity and virulence and resistance regulation in $A$. baumannii.

We characterized at the genetic and phenotypic levels two Acinetobacter baumannii isolates derived from the modern and broadly used parental AB5075 reference strain [13], received from two different laboratories, which exhibited different behaviors on solid media. For the first one, renamed AB5075-VUB (WT), we mostly observe opaque and mucoid colonies with occasional transient translucent and non-mucoid colonies, as previously described [10]. Whereas for the second one, named AB5075-VUBitrA::ISAba13, only stable translucent bacteria are observed. We sequenced and compared de novo assembled whole genomes for both strains (Supplementary Table. 1). The major difference detected within the capsule locus (KL25) is an ISAba13 Insertion Sequence (IS) interrupting the itrA gene of the itrA::ISAba13 mutant (Fig. 1a and Supplementary Table. 2). Duplicated Target Repeats (DTR) of 9 nucleotides are found at both extremities of the ISAba13 element. The itrA gene encodes for the initial glycosyltransferase A required for both capsule assembly and O-linked protein glycosylation [14]. According to ItrA loss of function phenotypes [14], we predicted the insertion to inactivate itrA gene and therefore impair capsule production and virulence.

We used transmission electron microscopy (TEM) combined with capsule staining to directly visualize the exopolysaccharide capsule in the WT and itrA::ISAba13 strains. This assay confirms the production of a thick capsule by WT cells and the absence of capsule surrounding itrA::ISAba13 bacteria (Fig. 1b). To assess capsule formation in a fast and semi-quantitative way, we implemented a density gradient based method for $A$. baumannii bacteria $[15,16]$. This density gradient confirms the phenotypes observed in TEM (Fig. 1c and Supplementary Fig. 1). We generated the deletion and complementation strains that validate the phenotype observed and its direct link to the disruption of the itrA coding sequence. The itrA gene was cloned at the neutral attTn7 site under the control of a constitutive promoter [17] (Fig. 1c and Supplementary Table. 1). Opacity of colonies correlates with production (opaque phenotype) and lack (translucent phenotype) of capsule (Fig. 1c). The WT and itrA::ISAba13 mutant have similar growth in liquid medium (Supplementary

\footnotetext{
${ }^{1}$ Microbial Resistance and Drug Discovery, VIB-VUB Center for Structural Biology, VIB, Flanders Institute for Biotechnology, Brussels, Belgium. ${ }^{2}$ Structural Biology Brussels, Vrije Universiteit Brussel (VUB), Brussels, Belgium. ${ }^{3}$ Research Unit in the Biology of Microorganisms (URBM), NARILIS, University of Namur (UNamur), Namur, Belgium. ${ }^{4}$ Neuromics Support Facility, VIB Center for Molecular Neurology, VIB, Antwerp, Belgium. ${ }^{5}$ Department of Biomedical Sciences, University of Antwerp, Antwerp, Belgium. ${ }^{6}$ Department of Ecology and Evolution, University of Lausanne, Lausanne, Switzerland. ${ }^{\circledR}$ email: charles.vanderhenst@vub.vib.be
}

Received: 31 August 2021 Revised: 7 December 2021 Accepted: 16 December 2021

Published online: 23 December 2021 
a

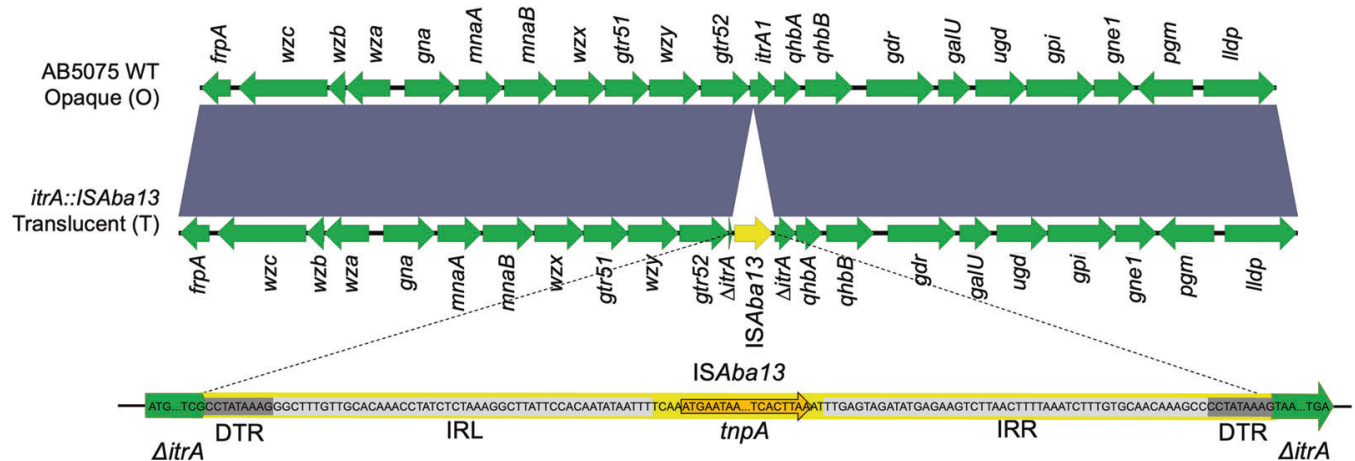

b

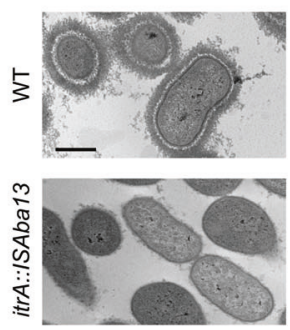

C

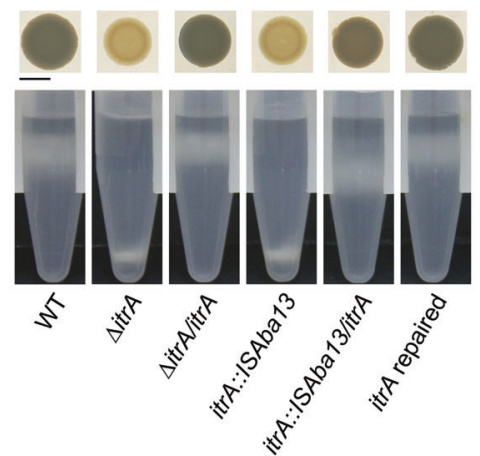

d

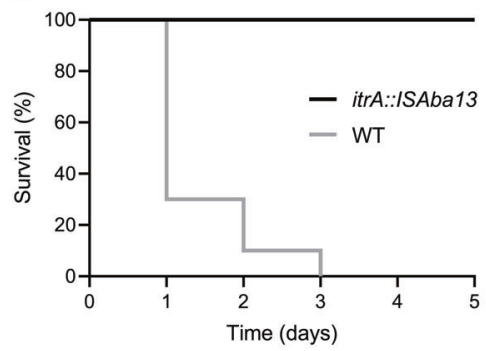

Fig. 1 Comparison of the WT and itrA::ISAba13 strains. a Sequence alignment of the K-loci of the WT and itrA::ISAba13 mutant. ISAba13 (represented in yellow) interupts itrA gene. IRR/IRL: respectively, inverted repeat right and left, tnpA: transposase gene, DTR: Direct Target Repeats of 9 nucleotides flanking ISAba13. Dark gray color indicates $100 \%$ sequence identity. b TEM of the WT and itrA::ISAba13 strains. Scale bar: $500 \mathrm{~nm}$. c Macrocolonies and density gradients. Scale bar: $0.5 \mathrm{~cm}$. d Survival of G. mellonella. Y axis: survival of larvae (\%), $x$ axis: time postinoculation (days), 10 larvae were infected for each condition and experiments were performed in biological duplicates.

Fig. 2a, b), even though we systematically observe a significantly reduced area for the macrocolonies of the itrA::ISAba13 strain compared to the WT strain on solid media (Supplementary Fig. 3 ).

We next assessed the in vivo virulence of the different strains using Galleria mellonella larvae [18]. We confirm the WT as fully virulent, all the larvae being killed after three days postinoculation, whereas all the larvae survive with the avirulent itrA::ISAba13 mutant after 5 days post inoculation (Fig. 1d). As controls, artificial deletion of itrA $(\triangle i t r A)$ in the WT strain abolishes virulence and capsule formation whereas complementation or in situ reparation of itrA in the itrA::ISAba13 mutant restores the virulence phenotypes (Supplementary Fig. 4).

The natural itrA::ISAba13 mutant is stably deficient for capsule production and associated virulence under rich laboratory conditions. Since the lack of capsule in various stresses and infectious conditions can be deleterious for bacteria [19], we assessed the stability of the itrA::ISAba13 phenotype. The presence of identical DTR flanking both ISAba13 extremities suggests that a scarless excision is possible, therefore reconstructing a WT and functional itrA gene copy [20]. However, for the translucent itrA:: ISAba13 strain, we do not detect any opaque colonies on solid rich media on a total of $2.8 \times 10^{5}$ colonies monitored. In addition, the colonies remain translucent after 4 successive passages in liquid media (Supplementary Fig. 5). As the liquid cultures are done in a rich medium, optimized for bacterial growth with minimal stress exposure, we decided to assess the stability of the phenotype in more stressful conditions. We inconsistently detect very rare opaque revertant clones in bacterial lawns produced by the itrA:: ISAba13 strain after 6 days of incubation on saturated solid medium $\left(2.5 \times 10^{4}\right.$ colonies counted in total) while no opaque colony are detected for the non-reversible $\triangle i t r A$ deletion strain $\left(5.1 \times 10^{4}\right.$ colonies counted in total), showing that natural reversion events can occur, but at very low frequency in the tested conditions (Supplementary Table. 3). We then used polymyxins, which are last resort antibiotics against multidrugresistant $A$. baumannii infections [21], as additional selective pressure. Moreover, bacterial capsule is involved in the resistance to these antibiotics [22]; while in a recent study, antibiotic treatment on $A$. baumannii biofilms correlates with a strong activity of ISAba13 [23]. We tested E-test strips (Biomérieux) of polymyxin B and colistin deposited on itrA::ISAba13 bacterial lawns. Under these conditions, we consistently detected opaque revertant clones after restriking bacteria surrounding the inhibition zone, with both antibiotics (Fig. 2a). Controls rule out any contamination possibility with a WT strain (Supplementary Fig. 6) and in addition, the $\triangle i t r A$ artificial deletion strain, lacking the reversion ability, does not generate any opaque clones (Fig. 2a). $\mathrm{PCR}$ and sequencing of the itrA coding sequence on both colistin (Revertant $\mathrm{CO}$ ) and polymyxin B (Revertant PO-B) revertants confirm the excision of the ISAba13 element and that the WT coding sequence is completely restored in a scarless manner (Fig. 2b and Supplementary Fig. 7). Accordingly, capsule production (Fig. 2c) and virulence (Fig. 2d) are restored to the WT level for the itrA::ISAba13 revertants. The fate of the ISAba13 insertion sequence after excision remains to be determined. After showing that reversion is qualitatively possible, we determined that it is a low frequency process (Supplementary Table. 3). The precise signal(s) regulating the occurrence and the frequency of both the integration and excision events remain(s) to be identified.

The reversion mechanism described in our study differs from the previously identified transient phenotypic phase variation [12]. The stability of the non-capsulated itrA::ISAba13 phenotype (Supplementary Fig. 5, 8, 9) can be beneficial for the bacterial population to survive infection by bacteriophages targeting the exopolysaccharide capsule of $A$. baumannii, against which noncapsulated bacteria were already shown to resist their predation 
a

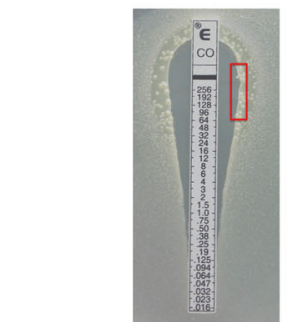

b

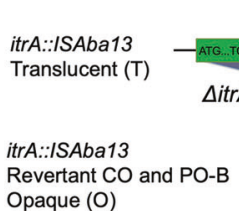

C

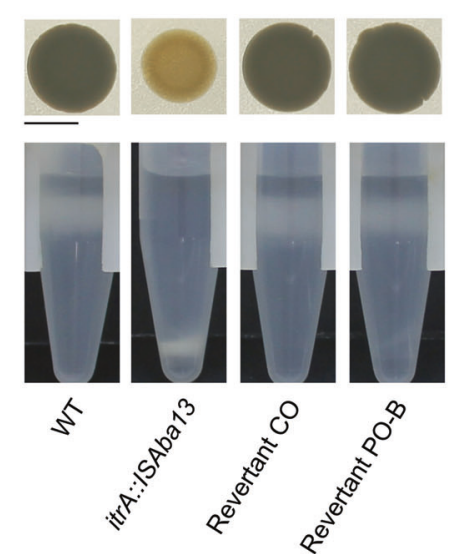
$\triangle$ itrA
itrA::ISAba13

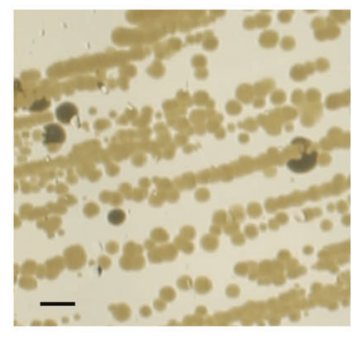

DTR

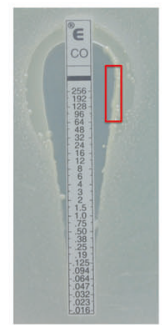

ISAba13
$\Delta i t r A$

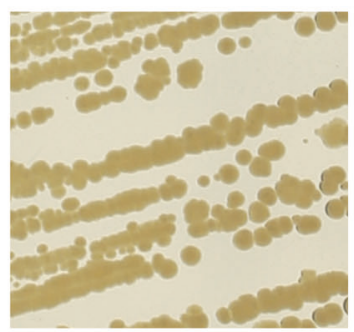

DTR

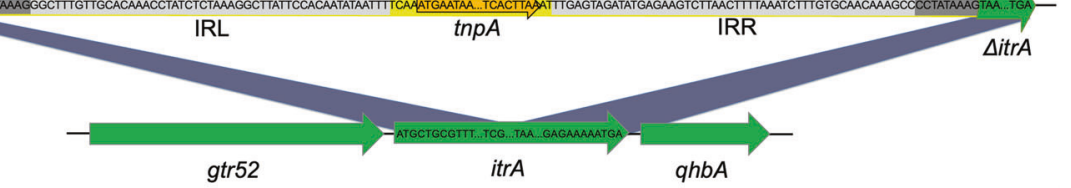

d
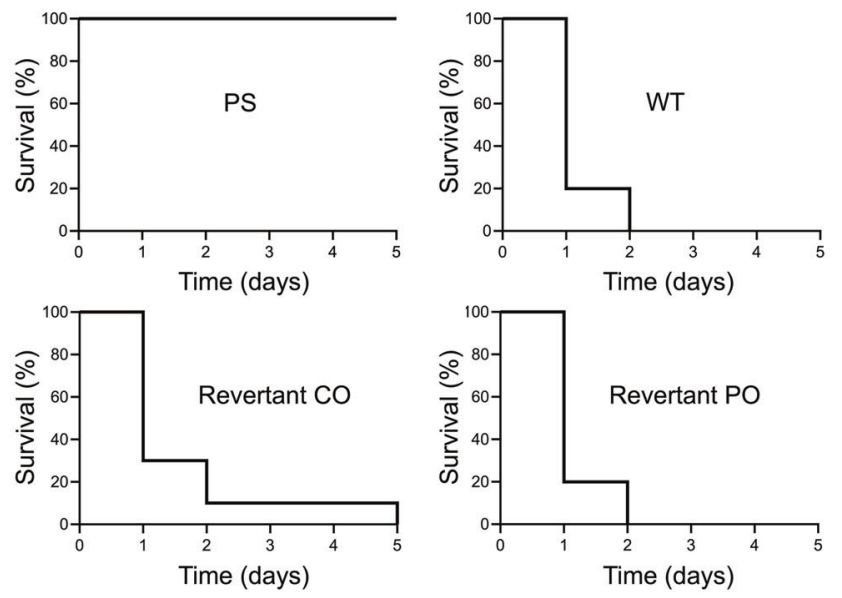

Fig. 2 ISAba13 scarless excision. a Opaque colonies isolated by plating itrA::ISAba13 bacteria harvested near the inhibition zone of a Colistin Etest. Bacteria near the inhibition zone (red square on left panel) were collected with a plastic loop and re-isolated on solid medium (right panel). The non-reversible control $\Delta i t r A$ strain shows $100 \%$ of translucent colonies. Scale bar: $0.2 \mathrm{~cm}$. b Sequence alignment of the K-loci of the itrA::ISAba13 natural mutant strain and Revertant CO and PO-B. c Macrocolonies phenotype on solid media and density gradients. Scale bar: $0.5 \mathrm{~cm}$. d Survival of $G$. mellonella over time. $Y$ axis: survival of larvae (\%), $x$ axis: time post-inoculation (days). 10 larvae were infected for each condition and experiments were performed in biological duplicates.

[24]. Therefore, the presence of a constant pool of sensitive preys sustaining the bacteriophage population is minimized compared to a higher frequency phenotypic phase variation. However, polysaccharide capsule plays key roles in other bacterial resistances and virulence. Hence, a reversion mechanism, such as the one here identified, is required to restore capsule formation, and the associated resistance and virulence abilities. Such reversion events, fostered by stress condition such as antibiotics, can represent a broad adaptative mechanism in microorganisms. A previous study described the modulation of cell surface sialic acid in Neisseria meningitidis by the insertion/excision of IS1301 (from the IS5 familly) in the siaA gene involved in capsule expression and endogenous LOS sialylation [25]. Concerning $A$. baumannii, out of 246 complete chromosome sequences available to date in GenBank, partial or complete insertion sequence within K-loci is identified in 23 isolates (9.31\%) (see Supplementary Table. 4), showing that IS elements can contribute to the locus dynamics and capsule regulation in $A$. baumannii. Moreover, ISAba13 IS are identified in a significant proportion (55 out of 246) of all the published genomes of $A$. baumannii, and several other IS elements are commonly present as potential key contributors to the genome dynamics [26].

Our study also highlights that every $A$. baumannii isolate exchanged deserves a careful and in-depth monitoring at the genetic level, using whole genome sequencing, along with a constant tracking of bacterial stocks.

\section{METHODS}

Bacterial strains and growth conditions

Bacterial strains and plasmids used in this study are listed in Supplementary Table. 1. Bacterial cultures were initiated from a single clone and were grown at $37^{\circ} \mathrm{C}$ in $5 \mathrm{ml}$ of liquid broth low salt ( $L B_{L S}$, Luria-Bertani formulation) from Duchefa Biochemie under agitation ( $166 \mathrm{rcf}$ ) or on solid LB-agar plates $(25 \mathrm{ml})$ unless indicated otherwise. $30 \mu \mathrm{g} / \mathrm{ml}$ of Apramycin sulfate salt (Sigma-Aldrich) or $10 \%$ of crystalized sucrose (Duchefa Biochemie) were used for the selection/counter selection of recombinant/ transformant clones. Four consecutive passages $(\sim 64 \mathrm{~h})$ were done in liquid culture to assess the phenotypical stability of the itrA::ISAba13 isolate as described in Supplementary Fig. 5. Eight hours and $16 \mathrm{~h}$ cultures were alternated twice and CFUs were plated from the initial bacterial culture and from the final passage. Quantification of height and area were measured using ImageJ. Statistical analyses were performed using unpaired $t$ test with GraphPad Prism 9. All experiments were carried out in biological triplicates unless stated otherwise.

\section{Genome assembly and K-loci comparison}

AB5075-VUB and itrA::ISAba13 are two clonally isolated strains originating from the parental AB5075 reference strain [13] (Supplementary Table. 1). 
They were called according to the field nomenclature, renaming the subcultured strains by adding "-VUB" to the strain name [27]. Their complete genomes were sequenced, de novo assembled and compared. Genomic DNA was extracted using the QIAGEN Genomic-tip 100/G following the manufacturer's recommendations. The short reads from MiSeq (lllumina) were trimmed for quality $(\mathrm{Q} \leq 20)$ and adaptor residues using Trimmomatic V0.36 [28] ( $\mathrm{Q} \leq 20$; read length $\leq 8$; sliding window 4:20). The long reads from MinION (Oxford Nanopore Technologies) were demultiplexed and basecalled using Guppy v3.2.2 (high accuracy model) and subsequently were adaptor and quality $(\mathrm{Q} \leq 8)$ trimmed using Porechop v0.2.2 (https://github. com/rrwick/Porechop) and BBDuk (https://sourceforge.net/projects/bbmap/) with default settings, respectively. The short reads were used to polish the long reads employing Ratatosk v0.7.0 [29] with default settings. The corrected long reads were then de novo assembled using Flye [30] v2.9 resulting in circular chromosomal contigs. The circular chromosomal contigs were polished using long reads via racon v1.4.20 (https://github.com/isovic/ racon) and Medaka v1.0.3 (https://github.com/nanoporetech/medaka) and subsequently using the short reads in two rounds of Pilon [31] v1.24 polishing. The mean coverage of short and long reads for AB5075-VUB was 41x and 340x and for AB5075-VUB-itrA::ISAba 13 the mean coverage was 37x and $490 \mathrm{x}$, respectively. The K-loci of the WT and itrA::ISAba13 were typed using Kaptive v0.7.3 (A. baumannii database downloaded in May, 2021), compared by pair-wise alignment in Geneious R9 (Biomatters, New Zealand) [32] and visualized in EasyFig [33] v2.2.2. The complete genomic sequences and corresponding sequencing data (including capillary sequencing data of amplicons) were deposited in GenBank under BioProject PRJNA701627. In order to verify the assemblies, the short and long reads were mapped to the region of itrA in AB5075-VUB (Supplementary Fig. 8) and to region of ISAba13 disrupting itrA in AB5075-VUB-itrA::ISAba13 (Supplementary Fig. 9). The visualization was performed in Geneious R9.

\section{Cloning and generation of mutant strains}

Plasmids and primers used to generate the strains used in this study are, respectively, listed in the Supplementary Table 1 and 5. Primers were purchased from Integrated DNA Technology (IDT) and sequencing was done using the Mix2Seq Kits - Overnight from Eurofins (Sanger sequencing). Gene deletion and complementation at the attTn7 site of AB5075-VUB were carried out following an adapted version of the previously published protocols [17] to obtain marker-less mutants. Complementation were done at the predicted neutral attTn7 site [34] and the genes were cloned under the influence of the artificial constitutive promoter $\mathrm{P}_{\text {strong }}$ (modified Ptac without lacO sites) and the RBS of the superfolder GFP (sfGFP) [17]. All constructs were introduced using natural transformation according to the previously published protocol [35]. Briefly, a large chimeric DNA fragment carrying the $s a c B$-aaC selection/counterselection cassette flanked by $1-2 \mathrm{~kb}$ homologous regions upstream and downstream of the targeted site was inserted at the locus of interest. The itrA coding sequence, the homologous regions flanking itr $A$ and the att $T n 7$ site were amplified by PCR from gDNA, the sfGFP coding sequence and $\mathrm{P}_{\text {strong }}$ promoter from the PASG-1 plasmid and the sacB-aaC cassette from the pMHL2 plasmid. PCR were performed using PrimeStarMax; TaKaRa (high fidelity DNA polymerase) and the resulting products were checked on agarose gel (1\%) and purified using the Wizard SV Gel and PCR CleanUp System (Promega) following the manufacturer's recommendations. The final chimeric DNA fragments were generated using the NEBuilder HiFi DNA Assembly Master Mix to sew the independent fragments. First the fragment containing the $s a c B-a a C$ cassette was introduced at the targeted locus and the recombinant strain selected on Apramycin $30 \mu \mathrm{g} / \mathrm{ml}$, was then transformed with a chimeric product containing the final desired fragment without any marker and counter selected on LB without $\mathrm{NaCl}(10$ $\mathrm{g} / \mathrm{L}$ tryptone, $5 \mathrm{~g} / \mathrm{L}$ yeast extract) - agar $10 \%$ sucrose and incubated $6 \mathrm{~h}$ at $30^{\circ} \mathrm{C}$.

\section{Colony morphology}

The colony morphology was assessed by spotting $5 \mu$ l of stationary phase bacteria from $\mathrm{O} / \mathrm{N}$ culture on $\mathrm{LB}_{\mathrm{LS}}$ agar plates containing a volume of $25 \mathrm{ml}$ of medium. After incubation (non-inverted) at $37^{\circ} \mathrm{C}$ for $24 \mathrm{~h}$, back light pictures of the petri dishes were taken with a Canon hand camera. Area of the macrocolonies were analyzed using ImageJ.

\section{Density gradient}

LUDOX Colloidal Silica ( $30 \mathrm{wt}$. \% suspension in $\mathrm{H}_{2} \mathrm{O}$, Merk) was used to semi-quantify capsule production of the different strains. The gradient solution was autoclaved before use and stored at $4{ }^{\circ} \mathrm{C} .1 \mathrm{ml}$ of $\mathrm{O} / \mathrm{N}$ culture was centrifuged for $5 \mathrm{~min}$ at $5000 \mathrm{rcf}$. The pellet was suspended in $1 \mathrm{ml}$ of PBS. $750 \mu \mathrm{l}$ of PBS resuspended bacteria were mixed with $250 \mu \mathrm{l}$ of LUDOX colloidal silica. This mix was then centrifuged for $30 \mathrm{~min}$ at $9000 \mathrm{rcf}$. Pictures were taken directly after the centrifugation using a Canon hand camera in front of black background.

\section{Transmission electron microscopy (TEM)}

Bacteria were fixed and stained according to the previously published protocol [10]. After the last treatment with the 1:1 mix of oxide propylene, the fixed pellet of bacteria was transferred into a cupule to be embedded in resin polymerized $12 \mathrm{~h}$ at $37^{\circ} \mathrm{C}, 48 \mathrm{~h}$ at $45^{\circ} \mathrm{C}$ and finally three days at $60^{\circ} \mathrm{C}$. The resin containing the bacteria was then cut into ultrathin sections ( 60 $\mathrm{nm})$, deposited on an electron microscopy grid and stained with acetate uranyl.

\section{Galleria mellonella infections}

Larvae were purchased from BioSystems Technology, TruLarv, stored at $15^{\circ} \mathrm{C}$ and used within the 5 days after arrival. Bacteria were washed twice in physiological saline (PS: $0.9 \% \mathrm{NaCl}$ in $\mathrm{H}_{2} \mathrm{O}$ ) solution and diluted to a concentration of $1.10^{7} \mathrm{CFU} / \mathrm{ml}$. Before injection, the larvae were incubated for $30 \mathrm{~min}$ at $4{ }^{\circ} \mathrm{C}$. $10 \mu \mathrm{l}$ of bacterial suspension in PS $\left(1 \times 10^{5} \mathrm{CFU} / \mathrm{ml}\right)$ were injected in the last left proleg of the larvae using a $0.3 \mathrm{ml}$ insulin syringes (BD MicroFine). 10 larvae were in inoculated per replicate. One control group was injected with PS as negative control and another group with the virulent AB5075-VUB WT as positive control of virulence. Survival was monitored every day over a 5 days period by checking the keratinization and mobility phenotypes. Infection experiments were done in biological duplicates, with a total of 20 larvae tested for each condition.

\section{Detection of phenotypical reversion}

To assess the itrA::ISAba13 phenotypic stability, we cultivated the bacteria in a dense lawn on solid LB medium for 6 days at $37^{\circ} \mathrm{C}$. Bacterial lawns were prepared by spreading $5 \mathrm{ml}$ of stationary phase bacteria from $\mathrm{O} / \mathrm{N}$ culture diluted to an OD of 1 (corresponding concentration $\sim 3 \times 10^{8}$ bacteria/ml). After $20 \mathrm{~s}$ the excess of liquid was removed, and the plates were dried and incubated at $37^{\circ} \mathrm{C}$ for 6 days. The $\triangle i t r A$ strain was used as a negative control of non-reversible phenotype. The itrA::ISAba13/sacB-aaC and itrA::ISAba13/sfGFP strains were used as additional controls, each of them carrying genetic markers, to show the absence of contamination by the marker-less WT strain. The presence or absence of ISAba13 in the itrA coding sequence was verified by PCR using the IS13V2_fW and IS13V2_rev primers (see Supplementary Table. 5). To assess the influence of antibiotics on the reversion events, similar bacterial lawns were prepared and Colistin Etest CO 256 WWB30 and Polymyxin B Etest purchased from Biomérieux were deposited on the dried bacterial lawn surface and incubated at $37{ }^{\circ} \mathrm{C}$ for 6 days. Bacteria were then collected from the inhibition edges and resuspended in PBS, diluted and plated on LB-agar. After $24 \mathrm{~h}$ of incubation at $37^{\circ} \mathrm{C}$, back light pictures of the petri dishes were taken using a Canon hand camera and colonies were screened for translucent (T) and opaque $(\mathrm{O})$ phenotypes. Opaque clones were isolated and ISAba13 insertion in itrA was screened by PCR, then sequenced to verify the itrA coding sequence.

\section{REFERENCES}

1. Weinberg SE, Villedieu A, Bagdasarian N, Karah N, Teare L, Elamin WF. Control and management of multidrug resistant Acinetobacter baumannii: a review of the evidence and proposal of novel approaches. Infect Prev Pr. 2020;2:100077.

2. Rice LB. Federal funding for the study of antimicrobial resistance in nosocomial pathogens: no ESKAPE. J Infect Dis. 2008;197:1079-81.

3. Tacconelli E, Carrara E, Savoldi A, Harbarth S, Mendelson M, Monnet DL, et al. Discovery, research, and development of new antibiotics: the WHO priority list of antibiotic-resistant bacteria and tuberculosis. Lancet Infect Dis. 2018;18:318-27.

4. US Department of Health and Human Services, CDC. Antibiotic resistance threats in the United States. Centers Dis Control Prev. 2019:1-113.

5. Harding CM, Hennon SW, Feldman MF. Uncovering the mechanisms of Acinetobacter baumannii virulence. Nat Rev Microbiol. 2018;16:91-102.

6. Geisinger E, Huo W, Hernandez-Bird J, Isberg RR. Acinetobacter baumannii: envelope determinants that control drug resistance, virulence, and surface variability. Annu Rev Microbiol. 2019;73:481-506.

7. Wyres KL, Cahill SM, Holt KE, Hall RM, Kenyon JJ. Identification of Acinetobacter baumannii loci for capsular polysaccharide $(\mathrm{KL})$ and lipooligosaccharide outer 
core $(\mathrm{OCL})$ synthesis in genome assemblies using curated reference databases compatible with kaptive. Microb Genomics. 2020;6:e000339.

8. Singh JK, Adams FG, Brown MH. Diversity and function of capsular polysaccharide in Acinetobacter baumannii. Front Microbiol. 2019;10:1-8.

9. Tipton KA, Rather PN. An omp-RenvZ two-component system ortholog regulates phase variation, osmotic tolerance, motility, and virulence in Acinetobacter baumannii strain AB5075. J Bacteriol. 2017;199:1-16.

10. Chin CY, Tipton KA, Farokhyfar M, Burd EM, Weiss DS, Rather PN. A highfrequency phenotypic switch links bacterial virulence and environmental survival in Acinetobacter baumannii. Nat Microbiol. 2018;3:563-9.

11. Anderson SE, Chin CY, Weiss DS, Rather PN. Copy number of an integronencoded antibiotic resistance locus regulates a virulence and opacity switch in Acinetobacter baumannii AB5075. mBio. 2020;11:1-19.

12. Ahmad I, Karah N, Nadeem A, Wai SN, Uhlin BE. Analysis of colony phase variation switch in Acinetobacter baumannii clinical isolates. PLoS ONE. 2019;14:1-13.

13. Jacobs AC, Thompson MG, Black CC, Kessler JL, Clark LP, McQueary CN, et al. $A B 5075$, a highly virulent isolate of Acinetobacter baumannii, as a model strain for the evaluation of pathogenesis and antimicrobial treatments. mBio. 2014;5:1-10.

14. Geisinger E, Isberg RR. Antibiotic modulation of capsular exopolysaccharide and virulence in Acinetobacter baumannii. PLoS Pathog. 2015;11:1-27.

15. Ardissone $S$, Fumeaux $C$, Bergé $M$, Beaussart $A$, Théraulaz $L$, Radhakrishnan $S K$, et al. Cell cycle constraints on capsulation and bacteriophage susceptibility. eLife. 2014;3:1-30.

16. Kon $\mathrm{H}$, Schwartz D, Temkin E, Carmeli $\mathrm{Y}$, Lellouche J. Rapid identification of capsulated Acinetobacter baumannii using a density-dependent gradient test. BMC Microbiol. 2020;20:1-11.

17. Godeux AS, Lupo A, Haenni M, Guette-Marquet S, Wilharm G, Maria-Halima Laaberki XC. Fluorescence-Based Detection of Natural Transformation in DrugResistant Acinetobacter baumannii. 2018;200:e00181-18.

18. Peleg AY, Jara S, Monga D, Eliopoulos GM, Moellering RC, Mylonakis E. Galleria mellonella as a model system to study Acinetobacter baumannii pathogenesis and therapeutics. Antimicrob Agents Chemother. 2009;53:2605-9.

19. Wen Z, Zhang JR. Bacterial capsules. Molecular Medical Microbiology: Second Edition. 2014. Elsevier Ltd.

20. Henderson IR, Owen P, James P. MicroReview Mol switches - Bact phase Var. 1999;33:919-32.

21. Chakravarty B. Genetic mechanisms of antibiotic resistance and virulence in Acinetobacter baumannii: background, challenges and future prospects. Mol Biol Rep. 2020;47:4037-46.

22. Olaitan AO, Morand S, Rolain JM. Mechanisms of polymyxin resistance: acquired and intrinsic resistance in bacteria. Front Microbiol. 2014;5:1-18.

23. Penesyan A, Nagy SS, Kjelleberg S, Gillings MR, Paulsen IT. Rapid microevolution of biofilm cells in response to antibiotics. npj Biofilms Microbiomes. 2019;5:34.

24. Gordillo Altamirano F, Forsyth JH, Patwa R, Kostoulias X, Trim M, Subedi D, et al Bacteriophage-resistant Acinetobacter baumannii are resensitized to antimicrobials. Nat Microbiol. 2021;6:157-61.

25. Hammerschmidt S, Hilse R, Van Putten JPM, Gerardy-Schahn R, Unkmeir A, Frosch $M$. Modulation of cell surface sialic acid expression in Neisseria meningitidis via a transposable genetic element. EMBO J. 1996;15:192-8.

26. Adams MD, Bishop B, Wright MS. Quantitative assessment of insertion sequence impact on bacterial genome architecture. Microb Genomics. 2016;2:e000062.

27. Gallagher LA, Ramage E, Weiss EJ, Radey M, Hayden HS, Held KG, et al. Resources for genetic and genomic analysis of emerging pathogen Acinetobacter baumannii. J Bacteriol. 2015;197:2027-35.

28. Bolger AM, Lohse M, Usadel B. Trimmomatic: a flexible trimmer for Illumina sequence data. Bioinformatics. 2014;30:2114-20.

29. Holley G, Beyter D, Ingimundardottir H, Møller PL, Kristmundsdottir S, Eggertsson $\mathrm{HP}$, et al. Ratatosk: hybrid error correction of long reads enables accurate variant calling and assembly. Genome Biol. 2021;22:1-22.

30. Kolmogorov M, Yuan J, Lin Y, Pevzner PA. Assembly of long, error-prone reads using repeat graphs. Nat Biotechnol. 2019;37:540-6.

31. Walker BJ, Abeel T, Shea T, Priest M, Abouelliel A, Sakthikumar S, et al. Pilon: an integrated tool for comprehensive microbial variant detection and genome assembly improvement. PLoS ONE. 2014;9. https://doi.org/10.1371/journal. pone.0112963.

32. Wyres KL, Wick RR, Gorrie C, Jenney A, Follador R, Thomson NR, et al. Identification of Klebsiella capsule synthesis loci from whole genome data. Micro Genomics. 2016;2:e000102.
33. Sullivan MJ, Petty NK, Beatson SA. Easyfig: a genome comparison visualizer. Bioinformatics. 2011;27:1009-10.

34. Kumar A, Dalton C, Cortez-Cordova J, Schweizer HP. Mini-Tn7 vectors as genetic tools for single copy gene cloning in Acinetobacter baumannii. J Microbiol Methods. 2010;82:296-300.

35. Wilharm G, Piesker J, Laue M, Skiebe E. DNA uptake by the nosocomial pathogen Acinetobacter baumannii occurs during movement along wet surfaces. J Bacteriol. 2013;195:4146-53.

\section{ACKNOWLEDGEMENTS}

We would like to thank Wouter De Coster for his help in bioinformatical analyses and the Electron Microscopy Facility of UNamur for their involvement in TEM imaging. We are also grateful to Prof. Xavier Charpentier and his team for their advice to genetically modify $A$. baumannii bacteria and for providing us the plasmids used in our study.

\section{AUTHOR CONTRIBUTIONS}

Experiments: CW, CP, $A B$ and CV. Data analyses: $A B, M V G$, JW and CV. Sequencings: MS and TDP. Bioinformatical analyses: AV, MS, TDP, IM and CV. Drafting of the manuscript: CW and CV.

\section{FUNDING}

This project was supported by the Flanders Institute for Biotechnology (VIB) and has received funding from the European Union's Horizon 2020 research and innovation program under the Marie Sklodowska-Curie grant agreement No 748032 (CV). AB is recipient of a PhD fellowship Strategic Basic Research of the Research Foundation Flanders (FWO, File number: 77258).

\section{COMPETING INTERESTS}

The authors declare no competing interests.

\section{ADDITIONAL INFORMATION}

Supplementary information The online version contains supplementary material available at https://doi.org/10.1038/s41396-021-01179-3.

Correspondence and requests for materials should be addressed to Charles Van der Henst.

Reprints and permission information is available at http://www.nature.com/ reprints

Publisher's note Springer Nature remains neutral with regard to jurisdictional claims in published maps and institutional affiliations.

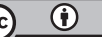

Open Access This article is licensed under a Creative Commons Attribution 4.0 International License, which permits use, sharing adaptation, distribution and reproduction in any medium or format, as long as you give appropriate credit to the original author(s) and the source, provide a link to the Creative Commons license, and indicate if changes were made. The images or other third party material in this article are included in the article's Creative Commons license, unless indicated otherwise in a credit line to the material. If material is not included in the article's Creative Commons license and your intended use is not permitted by statutory regulation or exceeds the permitted use, you will need to obtain permission directly from the copyright holder. To view a copy of this license, visit http://creativecommons. org/licenses/by/4.0/.

(c) The Author(s) 2022 